\title{
Apoyo social y resiliencia: factores protectores en cuidadores principales de pacientes en hemodiálisis
}

\author{
Lesnay Martínez Rodríguezㄹ, Evelyn Fernández Castilloㄹ, Elianys González Martínez ${ }^{1}$, Yunaisi de la C. Ávila \\ Hernández ${ }^{1}$, Amanda Lorenzo Carreiro ${ }^{3}$, Henry Luis Vázquez Morales ${ }^{2}$ \\ ${ }^{1}$ Universidad Central "Marta Abreu" de las Villas. Cuba \\ 2 Hospital "Arnaldo Milián Castro". Cuba \\ ${ }^{3}$ Universidad Central de las Villas. Cuba
}

Como citar este artículo:

Martínez-Rodríguez L, Fernández-Castillo E, González-Martínez E, Vázquez-Morales HL. Apoyo social y resiliencia: factores protectores en cuidadores principales de pacientes en hemodiálisis. Enferm Nefrol. 2019 Abr-Jun;22(2):130-9

\section{Resumen}

Introducción: Garantizar el cuidado del paciente en hemodiálisis se reconoce como una actividad compleja, exigente y estresante a la cual se expone de manera reiterada el cuidador. El apoyo social y la resiliencia constituyen factores protectores que permiten amortiguar el impacto del estrés en la salud mental de los cuidadores.

Objetivo: Analizar el apoyo social y la resiliencia como factores protectores en los cuidadores de pacientes en hemodiálisis.

Material y Método: Estudio mixto secuencial con alcance descriptivo. La selección muestral se realizó de forma intencional-no probabilística para una muestra de 67 cuidadores de pacientes en hemodiálisis asistentes al Servicio de tratamiento sustitutivo durante el 2018.

Se emplearon como instrumentos un Cuestionario de variables sociodemográficas, el Cuestionario de apoyo social funcional Duke-UNK-11 y la Escala reducida de Resiliencia Connor-Davidson. Se desarrollaron entrevistas grupales para la recogida de información cualitativa.

Resultados: La percepción de apoyo social así como las dimensiones de apoyo afectivo y confidencial se expresan en niveles medios y bajos, distinguiéndose la familia como la principal red de apoyo para los cuidadores. La

\section{Correspondencia:}

Universidad Central "Marta Abreu" de las Villas Facultad Ciencias Sociales. Departamento de Psicología Ctra. Camajuaní, Km 5 1⁄2. Santa Clara, Villa Clara. Cuba E-mail: lesnay@uclv.cu resiliencia alcanza niveles medio y alto siendo el optimismo, el sentido del humor y el establecimiento de relaciones sociales los principales mecanismos resilientes para enfrentar la actividad de cuidado.

Conclusiones: El apoyo social y la resiliencia se comportan como factores protectores en los cuidadores estudiados. Se constituyen como los principales recursos psicológicos amortiguadores del estrés que ofrecen una perspectiva diferente y enriquecedora para enfrentar situaciones adversas de manera efectiva en los cuidadores.

PALABRAS CLAVE: cuidador; apoyo social; resiliencia; hemodiálisis.

\section{Social support and resilience: protective factors in primary caregivers of patients on hemodialysis}

\section{Abstract}

Introduction: Ensuring the care in hemodialysis patient is a complex, demanding and stressful activity to which the caregiver is repeatedly exposed. Social support and resilience are protective factors that allow to mitigate the impact of stress on the caregiver's mental health.

Objective: Analyze social support and resilience as protective factors in caregivers of patients on hemodialysis.

Material and Method: A sequential mixed model design with descriptive scope. A non-probability sampling was made for a sample of 67 caregivers of hemodialysis pa- 
tients attending the Substitute Treatment Service during 2018.

A questionnaire of sociodemographic variables, the Duke-UNK-11 Functional Social Support Questionnaire and the Connor-Davidson Reduced Resilience Scale were used as instruments. Group interviews were developed for the collection of qualitative information.

Result: The perceptions of social support as well as the dimensions of affective and confidential support are expressed in medium and low levels, distinguishing the family as the main support network for caregivers. Resilience reaches medium and high levels, with optimism, a sense of humor and the establishment of social relationships as the main resilient mechanisms to approach the care activity.

Conclusions: Social support and resilience operate as protective factors in the caregivers studied. Both are considered as the main psychological resources to mitigate stress. Also they offer a different and enriching perspective to face adverse situations effectively in caregivers.

KEYWORDS: caregiver; social support; resilience; hemodialysis.

\section{Introducción}

El incremento de la insuficiencia renal crónica se debe al aumento de enfermedades crónicas degenerativas de gran prevalencia como la hipertensión arterial, diabetes mellitus, infecciones sistémicas y del tracto urinario, litiasis renal y enfermedades hereditarias ${ }^{1}$. La hemodiálisis se reconoce como una de las modalidades terapéuticas para la sustitución de la función renal que se realiza a partir de un procedimiento artificial apoyado por una máquina que dializa la sangre y la libera de las toxinas².

El diagnóstico de la enfermedad renal y su tratamiento sustitutivo genera como impactos en la familia la modificación en la estructura organizativa de sus miembros, la fragilidad y dependencia que experimentan los pacientes a nivel físico, emocional y social.

De acuerdo con la distribución de roles familiares las funciones recaen sobre el cuidador principal, quien se dedica de forma temporal o permanentemente al cuidado, custodia o protección de otra persona a la cual le falta o ha perdido autonomía física, psíquica o intelectual, y tiene necesidad de asistencia 0 ayuda para realizar actos corrientes de la vida diaria y, de modo particular, los relacionados con el cuidado personal ${ }^{3}$.

La asunción del rol implica para el cuidador conflictos, carencia de intereses personales, frustraciones, estados emocionales negativos, sobrecarga, insatisfacción de necesidades materiales o espirituales; pues la actividad de cuidado requiere no solo de disposición física sino también de un apoyo emocional hacia el familiar.

Se evidencian como principales variables asociadas al aumento de la carga del cuidador y relacionadas con el enfermo el grado de deterioro y/o pérdida de autonomía, comorbilidad con trastornos mentales de difícil manejo o la estabilidad hemodinámica. Por otro lado la edad, el sexo, el estilo de afrontamiento, la motivación para el cuidado, las redes de apoyo, los años de experiencia y las derivadas de la relación afectiva cuidador-enfermo tanto previas como actuales matizan el estado mental del cuidador ${ }^{4}$.

Las sensaciones de aislamiento social dependiendo de las circunstancias individuales en que se asume el rol de cuidador, constituye un cambio brusco si no existe una preparación y puede originar sentimientos de pérdida a partir de las expectativas personales o profesionales trazadas 5 .

Dentro del proceso salud-enfermedad el apoyo social se concibe como un recurso que facilita el éxito y actúa como variable mediadora en el proceso de estrés de las personas cuidadoras; desempeñándose como factor protector para la salud, facilitando el afrontamiento y la adaptación ante este tipo de situaciones ${ }^{6}$. Se afirma que la influencia del apoyo social garantiza un aumento de la resistencia de las personas ante cambios significativos durante su vida, estresores diarios, crisis personales y en el momento de adaptación o ajuste a las enfermedades de carácter crónico ${ }^{7}$.

Por otro lado, la resiliencia se evidencia como un proceso que permite retomar algún tipo de desarrollo a pesar de la ocurrencia de una situación traumática. La resiliencia se establece como un proceso dinámico, que depende de factores internos (personales) y externos (contextuales), tanto de riesgo como protectores, donde ambas dimensiones interactúan entre sí para generar un mecanismo que hace posible darle continuidad al desarrollo 0 a algunos aspectos del mismo, a pesar de las circunstancias ${ }^{8}$.

Los factores protectores se reconocen como influencias que modifican, mejoran o alteran la respuesta de una 
persona a algún peligro que predispone a un resultado no adaptativo ${ }^{8}$. Entre las condiciones promotoras de resiliencia, se destacan el apoyo social, la evaluación de los estresores así como el estilo de afrontamiento empleado por el cuidador'. Teniendo en cuenta el valor mediador que alcanzan los factores protectores en el bienestar de los cuidadores la investigación asumió como objetivo general: Analizar el apoyo social y la resiliencia como factores protectores en los cuidadores de pacientes en hemodiálisis. Se declaran como objetivos específicos del estudio:

- Identificar los niveles, tipos y fuentes de apoyo social en los cuidadores de pacientes hemodializados

- Determinar los niveles y mecanismos de resiliencia que poseen los cuidadores de pacientes hemodializados.

\section{Material y Método}

\section{Diseño}

Se realizó un estudio mixto secuencial con alcance descriptivo. El diseño de investigación se organizó mediante dos fases: en una primera etapa, se obtuvieron los datos cuantitativos que condujeron a la fase de análisis de datos cualitativos.

\section{Contexto de estudio}

El estudio se desarrolló durante el primer semestre del 2018 en los Servicios de Hemodiálisis del Hospital Docente "Arnaldo Milián Castro" de la ciudad de Santa Clara y el Hospital General de Caibarién ambos pertenecientes a la provincia de Villa Clara en Cuba. La investigación se desarrolló en los marcos de un convenio entre la institución hospitalaria y el Departamento de Psicología de la Universidad Central "Marta Abreu" de las Villas.

\section{Muestra}

Se incluyeron 67 cuidadores principales de pacientes en hemodiálisis a partir de un muestreo intencional no probabilístico. Se definieron criterios de inclusión, exclusión y salida para el proceso de selección muestral.

- Criterios de inclusión: Persona que se desempeñe como cuidador principal del paciente en tratamiento por hemodiálisis en Villa Clara. Asistencia en el horario diurno de tratamiento y la participación voluntaria en la investigación ofreciendo el consentimiento informado.
- Criterios de exclusión: Discapacidad intelectual y dificultades para la comprensión y realización de las pruebas. Persona o familiar que cumpla las funciones de acompañamiento al tratamiento.

- Criterios de salida: Abandono de la investigación debido al fallecimiento de su familiar o recibir trasplante y el no ofrecimiento del consentimiento informado.

\section{Aspectos éticos}

Se solicitó a las autoridades del Servicio de Hemodiálisis y del Hospital Docente "Arnaldo Milián Castro" el consentimiento para iniciar el estudio siendo aprobada su ejecución por el Consejo Científico y ético de la institución. Resultó imprescindible la firma del consentimiento informado por parte de los participantes posteriores a la explicación de forma individual sobre los objetivos y alcances de la investigación, respetando la participación voluntaria y confidencial.

\section{Técnicas}

- Cuestionario de variables sociodemográficas del cuidador: El instrumento agrupa de manera organizada los indicadores de las variables implicadas como objeto de estudio: edad, sexo, nivel de escolaridad, estado civil, vínculo laboral, grado de parentesco familiar-cuidador. El procesamiento de los datos se realizó de forma estadística mediante frecuencias descriptivas.

- Cuestionario de apoyo social funcional DukeUNK-11 ${ }^{10}$ : Evalúa el apoyo social percibido y consta de 11 ítems con una escala de respuesta tipo Likert para un rango de 1 a 5 . El rango de puntuación oscila entre 11 y 55 puntos y la puntuación obtenida refleja el apoyo percibido y las dimensiones de apoyo confidencial y apoyo afectivo.

- Escala reducida de Resiliencia Connor-Davidson ${ }^{11}$ : La variante reducida (CD-RISC10) validada para la población cubana mide la resiliencia de forma global y se evalúa mediante la sumatoria de los ítems. La escala consta de 10 ítems que se responden en una escala de tipo Likert para un rango de 0 a 4.

- Entrevista grupal: Se reconoce como una técnica dirigida a explorar los contenidos individuales en su expresión grupal amplificada. Para el estudio se conformaron dos grupos de 8 cuidadores teniendo como criterios la calidad, representatividad y distribución institucional de los participantes de acuerdo a las se- 
siones de tratamiento de sus familiares (Tabla 1). Se empleó la entrevista grupal de discusión pues en relación a un tema portador de sentido y mediante un contexto facilitador de la comunicación, actúa como inductor para la expresión plena de los participantes. (Tabla 2). Las sesiones de trabajo se realizaron en la sala de acompañantes del Servicio de Hemodiálisis con una duración máxima de 60 minutos. Para la transcripción de la información obtenida se utilizó solo como recurso la audiograbación pues los sujetos se negaron a ser grabados en vivo.

Tabla 1. Distribución de participantes en las entrevistas grupales.

\begin{tabular}{|l|c|c|c|}
\hline & Variables & Grupo 1 & Grupo 2 \\
\hline Edad & $25-37$ & 3 & 3 \\
(años) & $38-50$ & 4 & 2 \\
& $51-63$ & 1 & 3 \\
\hline Nivel & Secundaria Básica & 4 & 2 \\
de escolaridad & Bachiller & 2 & 3 \\
& Universitario & 2 & 3 \\
\hline Estado civil & Soltero & 2 & 1 \\
& Casado & 3 & 4 \\
& Divorciado & 3 & 3 \\
\hline Tiempo & 1 a 2 & 2 & 2 \\
como cuidador & 3 a 4 & 1 & 3 \\
(años) & 5 a 6 & 5 & - \\
& 7 a 8 & - & 3 \\
\hline
\end{tabular}

Tabla 2. Guía de preguntas de entrevistas grupales.

¿Cuáles son sus antecedentes y experiencias previas de cuidado?

¿Cómo describiría los modos de relación y convivencia con su familiar?

¿Qué particularidades caracterizan el cuidado a un paciente en hemodiálisis?

¿Cómo describiría su experiencia de cuidado? ¿Qué beneficios y desventajas han provocado en su vida?

¿De qué tipos o fuentes de apoyo se benefician? ¿Cuál es su nivel de satisfacción con el apoyo que perciben o reciben?

¿Qué recursos o mecanismos emplea para manejar de forma positiva el cuidado?

¿En qué espacios o momentos establecen relaciones con miembros de otros grupos?

¿Qué tipo de contactos (relaciones de amistad, de pareja) funcionan como sistema de apoyo para usted?

¿Con qué frecuencia disfruta de actividades sociales que no se relacionen con el cuidado?

\section{Procedimiento de investigación}

Para la recolección de la información en la fase cuantitativa se elaboró una base de datos para el procesamiento estadístico mediante el programa SPSS versión 22. Se realizaron análisis descriptivos y de frecuencia. Se empleó el análisis de contenido como método para el procesamiento de los datos cualitativos a partir de indicadores (Figura 1). Se contrastaron los datos obtenidos en la fase cuantitativa a través de entrevistas grupales. Las verbalizaciones de los cuidadores se codificaron a través de la letra $\mathrm{C}$ mayúscula acompañada con un número en subíndice en correspondencia con sus intervenciones durante las entrevistas.

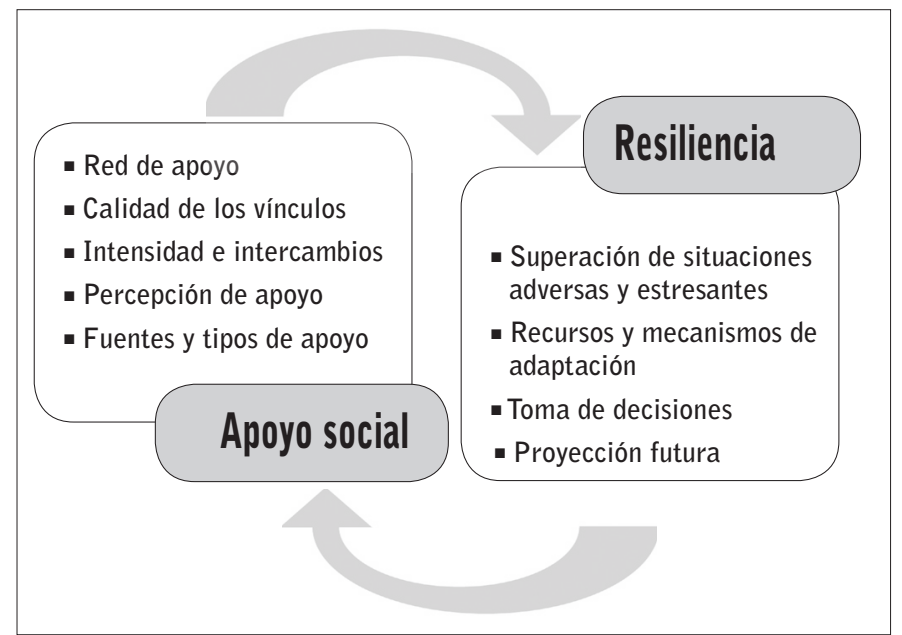

Figura 1. Indicadores cualitativos de análisis de los datos.

Se evidencia un predominio del género femenino en la asunción del rol para un $71,6 \%$ de la muestra con respecto al $28,4 \%$ de los cuidadores pertenecientes al género masculino. Las edades oscilan en el rango de 25 a 76 años de edad, con un predominio de cuidadores en las edades comprendidas entre 38 y 50 años que representan el $34,3 \%$ de los participantes. En relación al nivel de escolaridad se identifica que los cuidadores poseen formación técnico medio y nivel universitario para una distribución del 23,9\% en ambos casos ( Tabla 3$)$.

Se reconoce que el $59,7 \%$ (40) de la muestra no presentan vínculo laboral alguno y solo el $40,3 \%$ (27) se mantiene activo laboralmente. El 37,3\% (25) de la muestra valora que el abandono del vínculo laboral resultó como consecuencia de la tarea de cuidado de su familiar. En tanto el 25,4\% (17) no presentaba ningún 
Tabla 3. Distribución por edad, escolaridad, estado civil y tiempo de cuidado.

\begin{tabular}{lccc} 
& & & \\
& & & \\
Edad & & & \\
(años) & $25-37$ & 16 & 23,9 \\
& $38-50$ & 23 & 34,3 \\
& $51-63$ & 18 & 26,9 \\
Nivel de & $64-76$ & 10 & 14,9 \\
Escolaridad & Primario & 4 & 6,0 \\
& Secundaria Básica & 15 & 22,4 \\
& Técnico Medio & 16 & 23,9 \\
& Obrero calificado & 3 & 4,5 \\
Estado & Bachiller & 13 & 19,4 \\
civil & Universitario & 16 & 23,9 \\
& Soltero & 10 & 14,9 \\
& Casado & 43 & 64,2 \\
Tiempo & Unión consensual & 2 & 3,0 \\
como cuidador & Divorciado & 10 & 14,9 \\
(años) & Viudo & 1 & 1,5 \\
& 1 a 2 & 28 & 41,8 \\
& 3 a 4 & 12 & 17,9 \\
& 5 a 6 & 5 & 7,5 \\
& 7 a 8 & 3 & 4,5 \\
& 9 a 10 & 7 & 10,5 \\
& 11 a 12 & 2 & 3,0 \\
& 13 a 15 & 5 & 7,5 \\
& 18 a 23 & 3 & 4,5 \\
& 30 a 35 & 2 & 3,0 \\
\hline
\end{tabular}

vínculo laboral previo a sus funciones como cuidador. Se evidencia que el $41,8 \%$ (28) de los cuidadores ejecutan sus funciones por un periodo entre 1 a 2 años, sin embargo se identifican un rango máximo de 33 a 35 años para al menos 2 de los participantes (Tabla 3 ).

De acuerdo al grado de parentesco los datos más significativos encontrados fueron entre hijos y esposos que se encuentran desempeñando el rol de cuidador principal para un $40,3 \%$ (27) y un $50,7 \%$ (34) respectivamente.

Se constató que el apoyo social funcional de tipo confidencial en los cuidadores se expresa en un nivel medio para el $67,2 \%$ y el $32,8 \%$ percibe un nivel bajo. Por su parte el apoyo afectivo se ubica en el nivel medio para un $58,2 \%$ y solo el $14,9 \%$ de la muestra se ubican en el nivel alto. Se evidencia que los cuidadores perciben un mayor apoyo social funcional de tipo afectivo con respecto al apoyo confidencial (Gráfico 1).

Los cuidadores refieren que la familia constituye la principal fuente de apoyo compuesta por hijos-hijas, hermanos y esposo(a) y los amigos. Respecto a ello expresan: $C_{1}$ "generalmente es la familia quien nos apoya...", $\mathrm{C}_{5}$ "mis hijas me ayudan en las labores de la casa", $\mathrm{C}_{3}$ "tengo una familia que me apoya y me ayuda

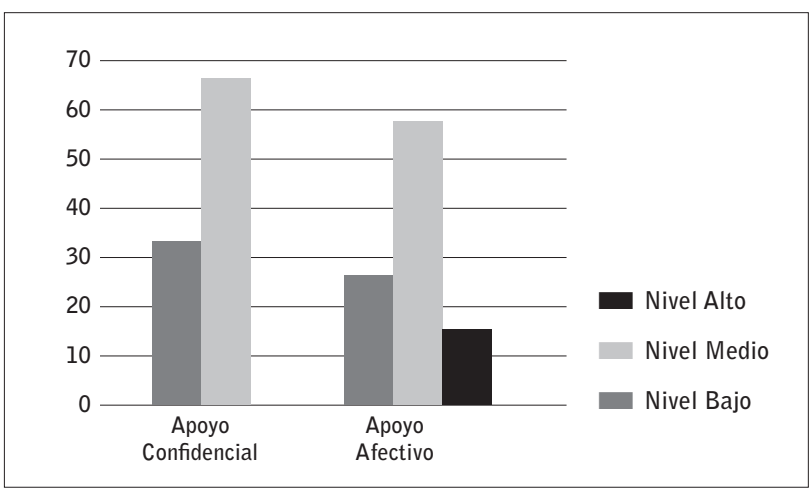

Gráfico 1. Niveles de apoyo social funcional.

en la medida de sus posibilidades", $\mathrm{C}_{4}$ "me siento apoyado porque tengo muy buenos amigos", $\mathrm{C}_{2}$ "somos una familia pequeña pero muy unida". Estas expresiones permiten comprender que los vínculos entre los cuidadores y sus fuentes de apoyo se establecen de manera favorable y que el apoyo instrumental se reconoce como un recurso para afrontar de forma saludable el proceso de cuidado.

Se identifica, sin embargo, una marcada carencia de apoyo afectivo y confidencial así como la necesidad por parte de los cuidadores de establecer un contacto más cercano y frecuente con los miembros de sus redes de apoyo. Este factor se evidencia de igual modo con el personal del hospital, reconociendo a la institución como una fuente de apoyo y señalan la necesidad de un mayor apoyo informativo por parte del personal médico. En relación a ello expresan: $C_{6}$ "los médicos pueden ayudarnos más, informarnos mejor", $\mathrm{C}_{7}$ "una enfermera que los trate bien salen más tranquilo y esto nos ayuda".

El personal médico, el servicio de transporte que el hospital proporciona y la ayuda económica de algunas instituciones gubernamentales se establecen como las principales fuentes de apoyo económico e institucional. Muchos de los cuidadores satisfacen la necesidad de apoyo afectivo en la propia relación con el familiar de cuidado expresada en verbalizaciones como: $\mathrm{C}_{2}$ "si él puede yo también", $\mathrm{C}_{6}$ "el paciente es nuestra red emocional", $C_{1}$ "mi familia es la que se encarga de brindarme cariño", $C_{5}$ "mi familiar es muy positivo $y$ me da fuerzas para seguir adelante".

Por su parte el grupo de cuidadores que asisten al turno se reconocen entre sí como una red de apoyo al afirmar: $C_{1}$ "nosotros somos una familia aquí", $C_{7}$ "nos ayudamos unos a otros lo mismo a acompañar a un paciente si el cuidador no se encuentra, asistirlo cuando 
termina el tratamiento, en lo que sea necesario", este aspecto se considera una potencialidad dentro del grupo de cuidadores estudiados. Los cuidadores declaran que a medida que avanza el tratamiento en hemodiálisis las fuentes de apoyo progresivamente desaparecen o disminuyen la intensidad de su relación, quedando el familiar sin otra ayuda que la proporcionada por el cuidador. Al respeto valoran que $\mathrm{C}_{8}$ "es una enfermedad muy larga, muchas personas nos abandonan en el camino", $C_{3}$ "en esta enfermedad tan larga la familia te apoya, pero después la gente va sacándose del rollo", $\mathrm{C}_{7}$ "esta vida de nosotros tiene muchos huecos", las verbalizaciones expresan el malestar emocional que genera la pérdida de apoyo familiar.

Con respecto a los niveles de resiliencia que experimentan los cuidadores no se identifican diferencias significativas entre los niveles altos y medios (Gráfico 2).

Algunos cuidadores expresan: $\mathrm{C}_{9}$ "vivimos muy tensos, con mucha preocupación y mucho estrés y eso es 10 que más me agota", $\mathrm{C}_{10}$ "los pacientes aquí están grave y necesitan mucha ayuda". Ante estos estados emocionales displacenteras las personas reconocen como principal factor protector el contar con algún tipo de apoyo social pues minimiza las situaciones de estrés y permite enfrentar los problemas de manera efectiva. Esto se evidencia al afirmar: $C_{11}$ "enfrento de manera positiva las situaciones porque tengo muchas personas que me ayudan en este problema", $\mathrm{C}_{13}$ "me apoyan mucho en lo que me haga falta y a la hora que sea", $\mathrm{C}_{16}$ "recibo mucho apoyo de todo el mundo y esto me impulsa a seguir adelante".

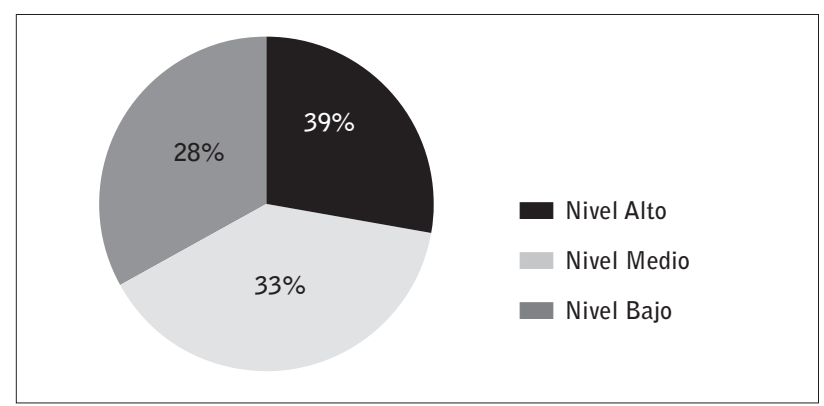

Gráfico 2. Niveles de resiliencia.

Los cuidadores identifican como sus principales fortalezas la asunción de una actitud optimista, el sentido del humor así como el mantenimiento de sus relaciones sociales. Con respecto a ello verbalizan $C_{15}$ "estar con otras personas en nuestra misma situación nos permite conversar, hacer chistes y pasar el tiempo de espera, aunque no dejamos de preocuparnos nunca", $C_{11}$ "si no fuera por estos buenos ratos nuestra vida sería un desastre", $\mathrm{C}_{14}$ "mientras estamos 4 horas en la Sala, al menos poder conversar con otros sobre nuestros problemas, eso ayuda". Estos elementos permiten afirmar que el grupo de cuidadores se establece como una valiosa fuente de apoyo social y compensan las situaciones estresantes a pesar de los efectos negativos provenientes de las funciones de cuidado.

En torno a la toma de decisiones los cuidadores alegan que no representan en sí mismo, un factor estresante sino una nueva alternativa o posibilidad de cambio: $C_{12}$ "soy una persona que no me cuesta tomar decisiones", $\mathrm{C}_{9}$ "cuando hay que hacer las cosas se hacen y ya", ello indica que la flexibilidad actúa como recurso resiliente en la toma de decisiones.

Por su parte, las responsabilidades asociadas al rol de cuidador implican una restructuración de proyectos de vida así como la modificación de rutinas, hábitos y estilos puedan interferir de algún modo con el desarrollo de su función y atención a su familiar. Se percibe entre los cuidadores una actitud pasiva y desalentadora ante la vida por lo que no se aprecia un proyecto de vida estructurado que regule y movilice su comportamiento en función de satisfacer sus necesidades, siendo por lo general subordinadas a las de su familiar. Esto se evidencia a partir de las siguientes verbalizaciones: $\mathrm{C}_{10}$ "Es mi hijo quien está en esta situación, por él haría cualquier cosa, tengo que ayudarlo, no queda otra alternativa que seguir ayudándolo hasta el final", $\mathrm{C}_{13}$ "soy padre del paciente y tengo que cuidarlo y luchar hasta que llegue el trasplante renal".

\section{Discusión y Conclusiones}

Los resultados obtenidos en relación a las variables sociodemográficas sexo, edad y nivel de escolaridad resultan coincidentes con estudios previos en cuidadores $^{12,13}$. La feminización del cuidado se reconoce como un fenómeno asociado a la función del rol y ampliamente contrastado en las investigaciones así como los altos niveles de escolarización en cuidadores garantizan mayor calidad en las funciones en correspondencia con el grado de formación académica ${ }^{12,13}$.

Aun cuando la mayor representación por edad se ubica en el rango de 38-51 años es preciso señalar que el $41,8 \%$ (28) de los cuidadores son adultos medios y mayores, lo cual demuestra que las ayudas se ofrecen 
por personas que a su vez requieren de atenciones y cuidado. El mantenimiento de una vida laboral resulta complicado para los cuidadores cuando se combina con la actividad de cuidado, proceso que se complejiza debido a los años en el desempeño del rol ${ }^{13}$.

Se evidencia que los cuidadores experimentan desgaste físico y emocional a partir de la sobrecarga de roles lo cual se agudiza debido a ineficientes redes de apoyo social que contribuyan con estas tareas ${ }^{12,15}$. El estudio afirma que el apoyo social constituye una necesidad en los cuidadores y un recurso que proporciona información, capacitación y por sí mismo apoyo al cuidador lo cual disminuye la sobrecarga y mejora el proceso de adquisición de habilidades para cuidar ${ }^{14}$. La convivencia con el cuidador garantiza en el paciente una mayor calidad de vida, bienestar emocional y se reconoce que una mayor percepción de apoyo minimiza los estados emocionales displacenteros, datos que afirman el valor de la familia como insustituible red de apoyo social ${ }^{15,16}$. Los resultados obtenidos confirman que la presencia de apoyo social positivo se expresa mediante muestras de afecto, escucha, consejo y comprensión.

El estudio corrobora que la familia constituye la principal fuente de apoyo social ${ }^{17}$, siendo una red capaz de ofrecer apoyo de forma incondicional tanto a pacientes como a cuidadores aun cuando se identifica la inestabilidad y poca permanencia de sus miembros. Con respecto a los tipos de apoyo social recibidos se evidencia que las principales necesidades de los cuidadores se relacionan con el apoyo instrumental, informativo y emocional.

La resiliencia actúa en los cuidadores como amortiguadores del proceso de cuidado y permite un control sobre el impacto negativo que genera la enfermedad crónica y sus consecuencias emocionales ${ }^{18}$. En los cuidadores estudiados se evidencian algunos de los elementos resilientes que modulan la percepción de estrés como el sentido del humor y el optimismo, factores que reducen los riesgos de salud y promueven la resiliencia ${ }^{19}$. Los datos de nuestro estudio permiten afirmar que el apoyo social es una condición promotora de resiliencia así como el estilo de afrontamiento al estrés centrado en los problemas se expresa en cuidadores con niveles altos de resiliencia9,12.

A partir de los resultados encontrados, podemos decir que Las investigaciones en población cuidadora muestran que con independencia del tipo de cuidado que se ofrezca o quien se beneficie de los mismos, las altera- ciones emocionales constituyen las principales consecuencias para quien asume el rol. El estrés que provoca la situación de cuidado deteriora progresivamente la calidad de vida y la estabilidad emocional del cuidador dejándolo desprovisto de estrategias para un afrontamiento efectivo. Los factores de protección como la resiliencia y el apoyo social se reconocen como los recursos sociales y psicológicos que pueden modificar la experiencia de cuidado y contribuir a un mejor bienestar.

\section{Agradecimientos}

A los cuidadores participantes en la investigación por su colaboración.

Al personal médico y asistencial de los Servicios de Hemodiálisis.

Recibido: 09-07-18

Revisado: 05-11-18

Modificado: 02-02-19

Aceptado: 15-02-19

\section{Bibliografía}

1. Vazquez I, Jofré R, López Gómez J. Calidad de vida en pacientes en Hemodiálisis. En: Tratado de Hemodiálisis. España: Editorial Jims; 2006;797- 810.

2. Páez A, Jofré M, Azpiroz C, De Bortoli M. Ansiedad y depresión en pacientes con insuficiencia renal crónica en tratamiento de diálisis. Universitas Psychologica. 2009;8(1):117-24.

3. Rosell Conde FA. Usted puede... Mejorar los cuidados del enfermo dependiente. Cuba: Editorial Científico-técnica; 2016.

4. Andrade Espín AM. Caracterización psicosocial de cuidadores informales de adultos mayores con demencia. Revista Cubana de Salud Pública. 2008;34 (3):1-11. 
5. Flores E, Rivas E, Seguel F. Nivel de sobrecarga en el desempeño del rol del cuidador familiar de adulto mayor con dependencia severa. Ciencia y Enfermería. 2012;18(1):29-41. [Consultado 12 abril 2019]. Disponible en: https://doi.org/10.4067/ s0717-95532012000100004.

6. Pearlin L, Mullan J, Semple S, Skaff M. Caregiving and the Stress Process: An Overview of Concepts and Their Measures.The Gerontologist. 1990;30(1): 583-94. https://doi.org/10.1093/geront/30.5.583.

7. Felman L, Goncalves L, Chacón Puignau G, Zaragoza J, Bagés N, Pablo D. Relaciones entre estrés académico, apoyo social, salud mental y rendimiento académico en estudiantes universitarios venezolanos. Univ Psychol. 2008;7(3):739-51.

8. Morelato G. Resiliencia en el maltrato infantil: aportes para la comprensión de factores desde un modelo ecológico. Revista de Psicología. 2011;29 (2) 201-23.

9. Crespo M, Fernández-Lansac V. Resiliencia en cuidadores familiares de personas mayores dependientes. Anales de Psicología. 2015;31(1):19-27.

10. Sotelo K. Validación del Cuestionario DUKEUNK-11 en cuidadores de pacientes en hemodiálisis. [Tesis de Diploma] Cuba: Universidad Central "Marta Abreu" de las Villas; 2018.

11. Rodríguez Martín B, Moleiro Pérez 0. Validación de instrumentos psicológicos: Criterios Básicos. Santa Clara: Feijóo; 2012. p.71-5.

12. Martínez Rodríguez L, Grau Valdés $Y$, Rodríguez Umpierre, R. Estrategias de afrontamiento al estrés en cuidadores de pacientes en hemodiálisis. Enferm Nefrol. 2017;20(2): 139-48. doi:http://dx.doi. org/10.4321/S2254-288420170000200007.
13. Lago Urbano R, Alós Villanueva P. Estudio descriptivo sobre el perfil de los cuidadores de personas con demencia: la feminización del cuidado. Psicogente. 2012;15(27):24-35. [Consultado 12 abril 2019] Disponible en: http://portal.unisimonbolivar.edu.co:82/rdigital/psicogente/index.php/ psicogente

14. Carreño-Moreno SP, Chaparro-Díaz L. Calidad de vida de los cuidadores de personas con enfermedad crónica. Aquichan. 2016;16(4):447-61. Doi: 10.5294/aqui.2016.16.4.4.

15. Arkerson GR Jr, Gutman RA. Health-related quality of life predictors of survival and hospital utilization. Health Care Financ Rev. 2000;21(3):171-84.

16. Kimmel PL. Psychosocial factors in adult end-stage renal disease patients treated with hemodialysis: Correlates and outcomes. Am J Kidney Dis. 2000;35(4):132-40.

17. Lin, N. A Network theory of social capital. 0xford University Press. 2005;1-25.

18. Böell JE, Silva DM, Hegadoren KM. Factores sociodemográficos y condicionantes de salud asociados a la resiliencia de personas con enfermedades crónicas: un estudio transversal. Revista Latino-Americana de Efermagem. 2016;1-9.

19. López-Fuentes I, Calvete E. Desarrollo del Inventario de factores de resiliencia ante la adversidad. Revista Ansiedad y estrés. 2016;22(2-3),110-17. [Consultado 12 abril 2019]. Disponible en: https:// doi.org/10.1016/j.anyes.2016.10.003. 


\section{Anexo 1. Cuestionario de apoyo social funcional Duke-UNK-11}

\section{Instrucciones:}

En la siguiente lista se muestran algunas cosas que otras personas hacen por nosotros o nos proporcionan. Elija para cada una la respuesta que mejor refleje su situación, según los siguientes criterios:

$1=$ Mucho menos de lo que deseo

$2=$ Menos de lo que deseo

$3=$ Ni mucho ni poco

$4=$ Casi como deseo

$5=$ Tanto como deseo

$\begin{array}{lllll}1 & 2 & 3 & 4 & 5\end{array}$

1.- Recibo visitas de mis amigos y familiares

2.- Recibo ayuda en asuntos relacionados con mi casa

3.- Recibo elogios y reconocimientos cuando hago bien mi trabajo

4.- Cuento con personas que se preocupan de lo que me sucede

5.- Recibo amor y afecto

6.- Tengo la posibilidad de hablar con alguien de mis problemas en el trabajo o en la casa

7.- Tengo la posibilidad de hablar con alguien de mis problemas personales y familiares

8.- Tengo la posibilidad de hablar con alguien de mis problemas económicos

9.- Recibo invitaciones para distraerme y salir con otras personas

10.- Recibo consejos útiles cuando me ocurre algún acontecimiento importante en mi vida

11.- Recibo ayuda cuando estoy enfermo en la cama 
Anexo 2. Escala reducida de Resiliencia

Instrucciones:

Por favor, indique cuál es su grado de acuerdo con las siguientes frases o caso durante el último mes. Si alguna en particular no le ha ocurrido, responda según crea que se hubiera sentido. Utilice para ello la siguiente escala:

$0=$ En absoluto

$1=$ Rara vez

$2=A$ veces

$3=\mathrm{A}$ menudo

4= Casi siempre

$\begin{array}{llll}0 & 1 & 2 & 3\end{array}$

4

Soy capaz de adaptarme cuando surgen cambios

Puedo enfrentarme a cualquier cosa

Cuando me enfrento a los problemas intento ver su lado cómico

Enfrentarme a las dificultades puede hacerme más fuerte

Tengo tendencia a recuperarme pronto luego de enfermedades, heridas u otras dificultades

Creo que puedo lograr mis objetivos, incluso si hay obstáculos

Bajo presión, me mantengo enfocado/a y pienso claramente

No me desanimo fácilmente ante el fracaso

Creo que soy una persona fuerte cuando me enfrento a los desafíos y

dificultades vitales

Soy capaz de manejar sentimientos desagradables/dolorosos:

Ej. tristeza, temor y enfado

Este artículo se distribuye bajo una Licencia Creative Commons Atribución-NoComercial 4.0 Internacional. https://creativecommons.org/licenses/by-nc/4.0/

Open Access (c) (7) \& 